\title{
Sur un exemple de Banach et Kuratowski
}

\author{
par \\ Robert Cauty (Paris)
}

\begin{abstract}
For $A \subset I=[0,1]$, let $\mathcal{L}_{A}$ be the set of continuous real-valued functions on $I$ which vanish on a neighborhood of $A$. We prove that if $A$ is an analytic subset which is not an $F_{\sigma}$ and whose closure has an empty interior, then $\mathcal{L}_{A}$ is homeomorphic to the space of differentiable functions from $I$ into $\mathbb{R}$.
\end{abstract}

1. Introduction et notations. Soit $\mathcal{C}$ l'espace des fonctions continues de $I=[0,1]$ dans $\mathbb{R}$ avec la topologie de la convergence uniforme. Pour tout sous-ensemble $A$ de $I$, soit $\mathcal{C}_{A}$ le sous-espace de $\mathcal{C}$ formé des fonctions s'annulant sur $A$, et soit $\mathcal{L}_{A}$ le sous-espace de $\mathcal{C}_{A}$ formé des fonctions qui s'annulent sur un voisinage de $A$. Banach et Kuratowski ont prouvé dans [1] que, si $A$ est un sous-ensemble non dense, analytique et non borélien de $I$, alors $\mathcal{L}_{A}$ est un espace coanalytique non borélien. Nous nous proposons ici de généraliser et de préciser ce résultat. Soit $\mathcal{D}$ le sous-espace de $\mathcal{C}$ formé des fonctions partout dérivables; c'est un exemple classique d'espace coanalytique non borélien. Nous prouverons le résultat suivant :

ThÉORÈme 1. Soit $A$ un sous-ensemble analytique non dense de I qui n'est pas un $F_{\sigma}$. Alors $\mathcal{L}_{A}$ est homéomorphe à $\mathcal{D}$.

Soit $2^{I}$ l'ensemble des fermés non vides de $I$ avec la topologie définie par la distance de Hausdorff. Pour tout sous-ensemble $A$ de $I$, soient $2_{A}^{I}$ le sous-ensemble de $2^{I}$ formé des fermés contenant $A$, et $\mathcal{N}_{A}$ celui formé des voisinages fermés de $A$. Banach et Kuratowski montrent aussi dans [1] que, si $A$ est un sous-ensemble non dense, analytique et non borélien de $I$, alors $\mathcal{N}_{A}$ est un espace coanalytique non borélien. Nous renforcerons ce résultat comme suit.

ThÉORÈme 2. Soit $A$ un sous-ensemble analytique non dense de I qui n'est pas un $F_{\sigma}$. Alors $\mathcal{N}_{A}$ est homéomorphe à $\mathcal{D}$.

1991 Mathematics Subject Classification: Primary 57N20. 
Les démonstrations de ces théorèmes reposent sur une caractérisation de $\mathcal{D}$ que nous avons développée dans [3]. Pour pouvoir la formuler, rappelons quelques définitions.

Si $f$ et $g$ sont deux fonctions de $Y$ dans $X$ et si $\mathcal{U}$ est un recouvrement ouvert de $X$, nous dirons que $f$ est $\mathcal{U}$-proche de $g$ si, pour tout $y$ dans $Y$, il y a un élément de $\mathcal{U}$ contenant à la fois $f(y)$ et $g(y)$. Un sous-ensemble $F$ d'un rétracte absolu de voisinage $X$ est appelé un $Z$-ensemble (resp. $Z$ ensemble au sens fort) dans $X$ s'il est fermé et si, pour tout recouvrement ouvert $\mathcal{U}$ de $X$, il existe une fonction continue $f$ de $X$ dans $X \mathcal{U}$-proche de l'identité et telle que $f(X) \cap F=\emptyset$ (resp. $\overline{f(X)} \cap F=\emptyset$ ). Une fonction $f: Y \rightarrow X$ est appelée un $Z$-plongement si c'est un plongement et si $f(Y)$ est un $Z$-ensemble dans $X$.

Notons $\mathcal{L}_{2}$ la classe des espaces coanalytiques. Un rétracte absolu de voisinage $X$ est dit $\mathcal{L}_{2}$-universel si, pour tout espace coanalytique $C$, toute fonction continue $f: C \rightarrow X$ et tout recouvrement ouvert $\mathcal{U}$ de $X$, il y a un $Z$-plongement $g: C \rightarrow X$ qui est $\mathcal{U}$-proche de $f$. $X$ est dit fortement $\mathcal{L}_{2}$-universel si, pour tout espace coanalytique $C$, tout fermé $D$ de $C$, toute fonction continue $f: C \rightarrow X$ dont la restriction à $D$ est un $Z$-plongement et tout recouvrement ouvert $\mathcal{U}$ de $X$, il existe un $Z$-plongement $g: C \rightarrow X$ qui est $\mathcal{U}$-proche de $f$ et vérifie $g|D=f| D$.

Nous avons prouvé dans [3] qu'un espace métrique séparable $X$ est homéomorphe à $\mathcal{D}$ si, et seulement si, il vérifie les trois conditions suivantes :

(I) $X$ est un rétracte absolu appartenant à $\mathcal{L}_{2}$,

(II) $X$ est réunion dénombrable de $Z$-ensembles au sens fort,

(III) $X$ est fortement $\mathcal{L}_{2}$-universel.

Si $x$ et $y$ sont deux points de $I$ et $B$ un sous-ensemble de $I$, nous posons

$$
d(x, y)=|x-y| \quad \text { et } \quad d(x, B)=\inf _{y \in B} d(x, y) ;
$$

nous notons $\delta(B)$ le diamètre de $B$. Nous supposons $2^{I}$ muni de la distance de Hausdorff $\varrho$ associée à $d$. Pour $f$ dans $\mathcal{C}$, nous posons $\|f\|=\sup _{t \in I}|f(t)|$.

Si $A$ est un sous-ensemble d'un espace $X$, une déformation instantanée de $X$ en $A$ est une homotopie $\varphi: X \times I \rightarrow X$ vérifiant $\varphi(x, 0)=x \forall x \in X$ et $\varphi(X \times] 0,1]) \subset A$. Il est connu que si $E$ est un sous-espace vectoriel partout dense d'un espace normé $X$, alors il existe une déformation instantanée de $X$ en $E$ (voir [10]).

2. Démonstration des théorèmes. Il faut montrer que $\mathcal{L}_{A}$ et $\mathcal{N}_{A}$ vérifient les conditions (I)-(III) de l'introduction. 
Vérification de (I). Les arguments de Banach et Kuratowski prouvent que $\mathcal{L}_{A}$ et $\mathcal{N}_{A}$ sont coanalytiques. Comme tout espace normé, $\mathcal{L}_{A}$ est un rétracte absolu; puisque $2_{A}^{I}$ est un rétracte absolu (en fait, d'après [6], une copie du cube de Hilbert), le lemme suivant achève la vérification de (I) (voir [10]).

Lemme 1. Il existe une déformation instantanée de $2_{A}^{I}$ en $\mathcal{N}_{A}$.

Démonstration. La fonction $\varphi: 2_{A}^{I} \times I \rightarrow 2_{A}^{I}$ définie par $\varphi(K, t)=$ $\{x \in I: d(x, K) \leq t\}$ est une telle déformation.

Remarquons que, $2_{A}^{I}$ étant compact, tout $Z$-ensemble dans $2_{A}^{I}$ est un $Z$-ensemble au sens fort; le lemme 1 et le lemme 2.6 de [3] entraînent alors que tout $Z$-ensemble de $\mathcal{N}_{A}$ est un $Z$-ensemble au sens fort.

Vérification de (II). Puisqu'il existe une déformation instantanée de $\mathcal{C}_{A}$ (resp. $2_{A}^{I}$ ) en $\mathcal{L}_{A}$ (resp. $\mathcal{N}_{A}$ ) et que tout $Z$-ensemble dans $\mathcal{C}_{A}$ ou $2_{A}^{I}$ est un $Z$-ensemble au sens fort, le lemme 2.6 de [3] entraîne qu'il suffit de montrer que $\mathcal{L}_{A}$ (resp. $\mathcal{N}_{A}$ ) est contenu dans une réunion dénombrable de $Z$-ensembles de $\mathcal{C}_{A}$ (resp. $2_{A}^{I}$ ). Fixons un point $t_{0}>0$ dans $A$; pour $n>1$, soit $A_{n}=A \cup\left[(1-1 / n) t_{0}, t_{0}\right]$.

Evidemment, $\mathcal{L}_{A}$ est contenu dans $\bigcup_{n=1}^{\infty} \mathcal{C}_{A_{n}}$ et les $\mathcal{C}_{A_{n}}$ sont fermés dans $\mathcal{C}_{A}$. Puisque $A$ est non dense, la fonction $\chi(t)=d(t, A)$ vérifie $\overline{\left.\left.\chi^{-1}(] 0,1\right]\right)}$ $=I$. Si $\varphi$ est une déformation instantanée de $\mathcal{C}_{A}$ en $\mathcal{L}_{A}$, on constate sans peine que la fonction $\psi: \mathcal{C}_{A} \times I \rightarrow \mathcal{C}_{A}$ définie par

$$
\psi(f, s)=\varphi(f, s)+s \chi
$$

est une déformation instantanée de $\mathcal{C}_{A}$ en $\mathcal{C}_{A} \backslash \bigcup_{n=1}^{\infty} \mathcal{C}_{A_{n}}$, ce qui entraîne que les $\mathcal{C}_{A_{n}}$ sont des $Z$-ensembles dans $\mathcal{C}_{A}$.

$\mathcal{N}_{A}$ est contenu dans $\bigcup_{n=1}^{\infty} 2_{A_{n}}^{I}$ et les $2_{A_{n}}^{I}$ sont fermés dans $2_{A}^{I}$. Si $\varphi$ est une déformation instantanée de $2_{A}^{I}$ en $\mathcal{N}_{A}$, on vérifie sans peine que la fonction $\psi: 2_{A}^{I} \times I \rightarrow 2_{A}^{I}$ définie par

$$
\psi(K, s)=\left(K \backslash\left[(1-s) t_{0}, t_{0}\right]\right) \cup \bar{A} \cup\left\{(1-s) t_{0}, t_{0}\right\}
$$

est continue et est une déformation instantanée de $2_{A}^{I}$ en $2_{A}^{I} \backslash \bigcup_{n=1}^{\infty} 2_{A_{n}}^{I}$, ce qui entraîne que les $2_{A_{n}}^{I}$ sont des $Z$-ensembles dans $2_{A}^{I}$.

Le lemme suivant, qui sera prouvé plus loin, est essentiel pour la vérification de (III).

Lemme 2. Si F est un sous-espace analytique d'un espace complet séparable $X$, alors il existe une fonction continue $\xi: X \rightarrow 2_{A}^{I}$ telle que $\xi^{-1}\left(\mathcal{N}_{A}\right)$ $=X \backslash F$. 
Vérification de (III) $\left(^{1}\right)$. Puisque tout $Z$-ensemble dans $\mathcal{L}_{A}$ ou $\mathcal{N}_{A}$ est un $Z$-ensemble au sens fort, il suffit, d'après la proposition 2.2 de [2], de montrer que tout ouvert $U$ de $\mathcal{L}_{A}\left(\right.$ resp. $\left.\mathcal{N}_{A}\right)$ est $\mathcal{L}_{2}$-universel. Soient $C$ un espace coanalytique, $F$ une fonction continue de $C$ dans $U$ et $\mathcal{U}$ un recouvrement ouvert de $U$. Nous pouvons supposer que $C$ est contenu dans le cube de Hilbert $Q=\prod_{n=1}^{\infty} I_{n}$, où $I_{n}=I$ pour tout $n$. D'après le lemme 2 , il existe alors une fonction continue $\xi: Q \rightarrow 2_{A}^{I}$ telle que

$$
\xi^{-1}\left(\mathcal{N}_{A}\right)=C \text {. }
$$

Prenons une fonction continue $\omega: U \rightarrow] 0,1]$ vérifiant

(2) quels que soient $x$ dans $U$ et $y$ dans $\mathcal{L}_{A}$ (resp. $\mathcal{N}_{A}$ ), si $\|x-y\| \leq 2 \omega(x)$ (resp. $\varrho(x, y) \leq 2 \omega(x)$ ), il y a un élément de $\mathcal{U}$ contenant à la fois $x$ et $y$.

Puisque $A$ est non dense et contient plus d'un point, nous pouvons trouver un point $a$ de $\bar{A}$ et un nombre $\eta>0$ tels que $[a, a+6 \eta]$ soit contenu dans $I$ et que $\bar{A} \cap] a, a+6 \eta]=\emptyset$. Nous avons alors

$$
d(t, A)=t-a \quad \text { pour } t \in[a, a+3 \eta] .
$$

Pour $\delta>0$, posons $N_{\delta}=\{t \in I: d(t, A) \leq \delta\}$ et $\stackrel{\circ}{N}_{\delta}=\{t \in I: d(t, A)$ $<\delta\}$.

Bien que les démonstrations pour $\mathcal{L}_{A}$ et $\mathcal{N}_{A}$ suivent le même plan, les détails techniques sont suffisamment différents pour nous obliger à distinguer les deux cas dans la suite.

- Cas de $\mathcal{L}_{A}$. Nous avons besoin de deux constructions auxiliaires.

Affirmation 1. Il existe une homotopie $\Phi: \mathcal{C}_{A} \times[0, \eta] \rightarrow \mathcal{C}_{A}$ vérifiant

$$
\begin{gathered}
\Phi(f, 0)=f \quad \forall f, \\
\Phi(f, \delta)(t)=0 \quad \text { si } t \in N_{\delta},
\end{gathered}
$$

(6) si $\left\{\left(f_{n}, \delta_{n}\right)\right\}_{n=1}^{\infty}$ est une suite de points de $\left.\left.\mathcal{C}_{A} \times\right] 0, \eta\right]$ telle que la suite $\left\{\Phi\left(f_{n}, \delta_{n}\right)\right\}$ converge vers un élément $g$ de $\mathcal{C}_{A}$ et que $\left\{\delta_{n}\right\}$ tende vers zéro, alors $\left\{f_{n}\right\}$ converge aussi vers $g$.

$\left({ }^{1}\right)$ La publication de cet article ayant été longuement retardée, est paru entretemps un résultat général ([4], théorème 3.1) permettant d'établir facilement l'universalité forte de $\mathcal{L}_{A}$. En effet, on peut déduire du lemme 2 que tout espace coanalytique est homéomorphe à un fermé de $\mathcal{L}_{A}$, et, si $J$ est un sous-intervalle de $I$ disjoint de $\bar{A}$, il est facile de vérifier que $\mathcal{L}_{A}$ est homéomorphe à $C(J) \times E \cong \mathbb{R}^{\infty} \times E$ où $E=\left\{f \in \mathcal{L}_{A}: f \mid J=0\right\}$. Le théorème 3.1 de [4] est donc applicable. Nous avons cependant maintenu la démonstration originale de (III), qui avait été conçue pour illustrer la généralité du plan de démonstration de l'universalité forte que nous utilisons, tout en mettant en évidence les nécessaires différences techniques. Notons d'ailleurs que la démonstration du théorème 3.1 de [4] suit aussi ce même plan. 
Nous pouvons définir $\Phi$ en posant $\Phi(f, 0)=f$ et, pour $\delta>0$,

- $\Phi(f, \delta)(t)=0$ si $\left.t \in N_{2 \delta} \backslash\right] a+\delta, a+2 \delta[$,

- $\Phi(f, \delta)(a+3 \delta / 2)=\sup \left\{f(t): t \in N_{3 \delta}\right\}$,

- $\Phi(f, \delta)$ est linéaire sur $[a+\delta, a+3 \delta / 2]$ et sur $[a+3 \delta / 2, a+2 \delta]$,

- $\Phi(f, \delta)(t)=f(t)$ si $t \notin \stackrel{\circ}{N}_{3 \delta}$,

- $\Phi(f, \delta)(t)=\frac{1}{\delta}(d(t, A)-2 \delta) f(t)$ si $2 \delta \leq d(t, A) \leq 3 \delta$.

Il résulte de (3) que ces conditions sont compatibles et que (5) est vérifiée. Il est clair que $\Phi$ est continue. Soit $\left\{\left(f_{n}, \delta_{n}\right)\right\}_{n=1}^{\infty}$ comme dans (6). Fixons $n \geq 1$ et soit $t$ un point de $I$. Si $d(t, A) \geq 3 \delta_{n}$,

$$
\left|f_{n}(t)-g(t)\right|=\left|\Phi\left(f_{n}, \delta_{n}\right)(t)-g(t)\right| \leq\left\|\Phi\left(f_{n}, \delta_{n}\right)-g\right\|,
$$

d'où $\left\|f_{n}-g\right\| \leq \max \left(\left\|\Phi\left(f_{n}, \delta_{n}\right)-g\right\|, \sup \left\{\left|f_{n}(t)\right|: t \in N_{3 \delta_{n}}\right\}+\sup \{|g(t)|\right.$ : $\left.t \in N_{3 \delta_{n}}\right\}$ ).

Par hypothèse, $\left\|\Phi\left(f_{n}, \delta_{n}\right)-g\right\|$ tend vers zéro. Puisque $g$ est continue et s'annule sur $A, \sup \left\{|g(t)|: t \in N_{3 \delta_{n}}\right\}$ tend vers zéro. Puisque $\{a+$ $\left.3 \delta_{n} / 2\right\}$ tend vers $a$, la convergence uniforme de $\Phi\left(f_{n}, \delta_{n}\right)$ vers $g$ garantit que $\sup \left\{\left|f_{n}(t)\right|: t \in N_{3 \delta_{n}}\right\}=\Phi\left(f_{n}, \delta_{n}\right)\left(a+3 \delta_{n} / 2\right)$ tend vers $g(a)=0$. Ceci montre que $\left\|f_{n}-g\right\|$ tend vers zéro.

Affirmation 2. Il existe une fonction continue $\Psi: Q \times] 0, \eta] \rightarrow \mathcal{C}_{A}$ vérifiant

$$
\begin{gathered}
\left.\left.\Psi^{-1}\left(\mathcal{L}_{A}\right)=C \times\right] 0, \eta\right], \\
\|\Psi(q, \varepsilon)\| \leq \varepsilon \quad \forall(q, \varepsilon) \in Q \times] 0, \eta], \\
\left.\left.\left.[\Psi(q, \varepsilon)]^{-1}(]-\infty, 0\right]\right)=\right] a+\varepsilon / 2, a+3 \varepsilon / 4[,
\end{gathered}
$$

(10) si $\Psi(q, \varepsilon)\left|[a+\varepsilon / 2, a+\varepsilon]=\Psi\left(q^{\prime}, \varepsilon\right)\right|[a+\varepsilon / 2, a+\varepsilon]$, alors $q=q^{\prime}$.

Pour construire $\Psi$, notons d'abord que la fonction $\bar{\xi}: Q \rightarrow \mathcal{C}_{A}$ définie par $\bar{\xi}(q)(t)=d(t, \xi(q))$ vérifie

$$
\begin{aligned}
0 \leq \bar{\xi}(q)(t) \leq 1 \quad \text { quels que soient } q \text { et } t, \\
\\
\xi^{-1}\left(\mathcal{L}_{A}\right)=C,
\end{aligned}
$$

la condition (12) résultant de (1). Pour $0<\varepsilon \leq \eta$, soit $\mu(\varepsilon)$ la fonction égale à 1 hors de $] a+\varepsilon / 3, a+3 \varepsilon$ [, à zéro sur $[a+\varepsilon / 2, a+2 \varepsilon]$ et linéaire sur $[a+\varepsilon / 3, a+\varepsilon / 2]$ et $\operatorname{sur}[a+2 \varepsilon, a+3 \varepsilon] ; \mu(\varepsilon)$ dépend continûment de $\varepsilon$.

Pour $n \geq 1$, soit $x_{n}=1 / 2+1 / 2^{n}$, et soit $y_{n}$ le milieu du segment $\left[x_{n+1}, x_{n}\right]$. Pour $q=\left(q_{n}\right)$ dans $Q$, définissons une fonction continue $\theta(q)$ : $I \rightarrow[-1,1]$ par les conditions

- $\theta(q)(0)=\theta(q)(1 / 2)=\theta(q)\left(x_{n}\right)=0(n \geq 1)$

- $\theta(q)(1 / 4)=-1$, 
- $\theta(q)\left(y_{n}\right)=q_{n} \cdot 2^{-n}(n \geq 1)$,

- $\theta(q)$ est linéaire sur chacun des segments [0,1/4], [1/4,1/2], $\left[x_{n+1}, y_{n}\right]$ et $\left[y_{n}, x_{n}\right], n \geq 1$.

Définissons alors $\Psi$ par

$$
\Psi(q, \varepsilon)(t)= \begin{cases}\varepsilon \mu(\varepsilon)(\bar{\xi}(q)(t)) & \text { si } t \notin] a+\varepsilon / 2, a+\varepsilon[, \\ \varepsilon\left[\theta(q)\left(\frac{2}{\varepsilon}\left(t-a-\frac{\varepsilon}{2}\right)\right)\right] & \text { si } t \in[a+\varepsilon / 2, a+\varepsilon] .\end{cases}
$$

On vérifie facilement que cette définition est cohérente et que $\Psi$ est continue. (7) résulte de (12) et du fait que $\Psi(q, \varepsilon)$ et $\varepsilon \bar{\xi}(q)$ sont égales sur $N_{\varepsilon / 3}$. (8) et (9) résultent de (11) et du choix de $\theta$. Enfin, pour $\varepsilon>0$ donné, si $\Psi(q, \varepsilon)\left|[a+\varepsilon / 2, a+\varepsilon]=\Psi\left(q^{\prime}, \varepsilon\right)\right|[a+\varepsilon / 2, a+\varepsilon]$, alors $\theta(q)=\theta\left(q^{\prime}\right)$, mais $\theta(q)\left(y_{n}\right)=\theta\left(q^{\prime}\right)\left(y_{n}\right)$ entraîne $q_{n}=q_{n}^{\prime}$ pour tout $n$, d'où (10).

Nous pouvons maintenant achever la démonstration dans le cas de $\mathcal{L}_{A}$. La condition (4) et la continuité de $\Phi$ nous permettent de trouver une fonction continue $\varepsilon: U \rightarrow] 0, \eta]$ vérifiant, pour tout $f \in U$,

$$
\begin{gathered}
\|f-\Phi(f, \varepsilon(f))\|<\omega(f), \\
\varepsilon(f)<\omega(f) .
\end{gathered}
$$

Posant $\widetilde{\varepsilon}(c)=\varepsilon(F(c)),(5)$ et (7) nous permettent de définir une fonction continue $G: C \rightarrow \mathcal{L}_{A}$ par

$$
G(c)=\Phi(F(c), \widetilde{\varepsilon}(c))+\Psi(c, \widetilde{\varepsilon}(c)) .
$$

Il résulte de (8), (13) et (14) que

$$
\|F(c)-G(c)\|<\omega(F(c))+\varepsilon(F(c))<2 \omega(F(c)),
$$

donc $F(c)$ et $G(c)$ appartiennent à un même élément de $\mathcal{U}$ d'après (2); en particulier, $G$ est à valeurs dans $U$.

Pour $t$ dans $[a, a+\widetilde{\varepsilon}(c)], G(c)(t)=\Psi(c, \widetilde{\varepsilon}(c))(t)$ d'après (5) et (3). Alors, (9) entraîne que $] a+\widetilde{\varepsilon}(c) / 2, a+3 \widetilde{\varepsilon}(c) / 4[$ est la composante de l'ensemble $G(c)^{-1}(]-\infty, 0[)$ qui suit immédiatement $a$, donc si $c$ et $c^{\prime}$ sont deux points de $C$ tels que $G(c)=G\left(c^{\prime}\right)$, alors $\widetilde{\varepsilon}(c)=\widetilde{\varepsilon}\left(c^{\prime}\right)$; en outre,

$$
\begin{aligned}
\Psi(c, \widetilde{\varepsilon}(c)) \mid[a+\widetilde{\varepsilon}(c) / 2, a+\widetilde{\varepsilon}(c)] & =G(c) \mid[a+\widetilde{\varepsilon}(c) / 2, a+\widetilde{\varepsilon}(c)] \\
& =G\left(c^{\prime}\right) \mid[a+\widetilde{\varepsilon}(c) / 2, a+\widetilde{\varepsilon}(c)] \\
& =\Psi\left(c^{\prime}, \widetilde{\varepsilon}(c)\right) \mid[a+\widetilde{\varepsilon}(c) / 2, a+\widetilde{\varepsilon}(c)],
\end{aligned}
$$

et (10) entraîne que $c=c^{\prime}$, ce qui montre que $G$ est injective.

Puisque $G$ est injective, pour prouver que c'est un plongement fermé dans $U$, il suffit de montrer que si $\left\{c_{n}\right\}_{n=1}^{\infty}$ est une suite de points de $C$ telle que la suite $\left\{G\left(c_{n}\right)\right\}$ converge vers un élément $g$ de $U$, alors $\left\{c_{n}\right\}$ a une sous-suite qui converge vers un point $c_{0}$ de $C$. Posant $\varepsilon_{n}=\widetilde{\varepsilon}\left(c_{n}\right)$, nous pouvons supposer que $\left\{\varepsilon_{n}\right\}$ tend vers $\varepsilon_{0} \in[0, \eta]$ et que $\left\{c_{n}\right\}$ tend vers un 
point $c_{0}$ de $Q$. Alors, $\varepsilon_{0}>0$. En effet, dans le cas contraire, $\left\{\Psi\left(c_{n}, \varepsilon_{n}\right)\right\}$ tendrait vers zéro d'après $(8)$, donc $\left\{\Phi\left(F\left(c_{n}\right), \varepsilon_{n}\right)\right\}$ tendrait aussi vers $g$; d'après (6), il en serait de même de $\left\{F\left(c_{n}\right)\right\}$ et, $\varepsilon$ étant continue, $\left\{\varepsilon_{n}\right\}$ tendrait vers $\varepsilon(g)>0$, ce qui est contradictoire.

La suite $\left\{\Psi\left(c_{n}, \varepsilon_{n}\right)\right\}$ tend vers $\Psi\left(c_{0}, \varepsilon_{0}\right)$. Puisque $\varepsilon_{0}>0$, nous pouvons supposer $\varepsilon_{n}>\varepsilon_{0} / 2$ pour tout $n$; d'après (5), $\Phi\left(F\left(c_{n}\right), \varepsilon_{n}\right) \mid N_{\varepsilon_{0} / 2}$ $=0$ pour tout $n$, donc, en passant à la limite, nous obtenons $g \mid N_{\varepsilon_{0} / 2}=$ $\Psi\left(c_{0}, \varepsilon_{0}\right) \mid N_{\varepsilon_{0} / 2}$. Puisque $g$ appartient à $\mathcal{L}_{A}, \Psi\left(c_{0}, \varepsilon_{0}\right)$ aussi, donc $c_{0}$ appartient à $C$ d'après $(7)$.

Reste à montrer que $G(C)$ est un $Z$-ensemble dans $U$. Pour cela, définissons d'abord une fonction $K:[0, \eta] \rightarrow \mathcal{L}_{A}$ par $K(0)=0$ et, pour $\delta>0$,

$$
K(\delta)(t)= \begin{cases}0 & \text { si } t \notin] a+\delta / 2, a+\delta[, \\ (t-a-\delta / 2) \sin \left(\frac{\delta \pi}{t-a-\delta / 2}\right) & \text { si } t \in] a+\delta / 2, a+\delta[.\end{cases}
$$

Il est clair que $K$ est continue. Définissons alors $\Lambda: \mathcal{L}_{A} \times[0, \eta] \rightarrow \mathcal{L}_{A}$ par

$$
\Lambda(f, \delta)=\Phi(f, \delta)+K(\delta) .
$$

D'après $(4), \Lambda(f, 0)=f$. En utilisant (5) et la définition de $K$, on constate que si $g=\Lambda(f, \delta)$ avec $\delta>0$, alors l'ensemble des composantes connexes de $\left.\left.\left(g^{-1}(]-\infty, 0[)\right) \cap\right] a, 1\right]$ n'a pas de plus petit élément, alors que nous avons remarqué plus haut que, pour $c$ dans $C,\left((G(c))^{-1}(]-\right.$ $\infty, 0])) \cap] a, 1]$ a pour composante minimale $] a+\widetilde{\varepsilon}(c) / 2, a+3 \widetilde{\varepsilon}(c) / 4[$. Par suite, $\left.\left.G(C) \cap \Lambda\left(\mathcal{L}_{A} \times\right] 0, \eta\right]\right)=\emptyset$; puisque $\Lambda(f, 0)=f$ pour tout $f \in \mathcal{L}_{A}$, cela entraîne que $G(C)$ est un $Z$-ensemble dans $U$.

- Cas de $\mathcal{N}_{A}$. Nous avons encore besoin de deux constructions auxiliaires.

Affirmation $\widetilde{1}$. Il existe une homotopie $\widetilde{\Phi}: 2_{A}^{I} \times[0, \eta] \rightarrow 2_{A}^{I}$ vérifiant

$$
\begin{gathered}
\widetilde{\Phi}(K, 0)=K \quad \forall K \in 2_{A}^{I}, \\
\text { pour } \varepsilon>0, \widetilde{\Phi}(K, \varepsilon) \backslash A \text { est fini. }
\end{gathered}
$$

En effet, d'après [6], il existe une homotopie $\varphi: 2^{I} \times[0, \eta] \rightarrow 2^{I}$ telle que $\varphi(K, 0)=K$ pour tout $K$ et que $\varphi(K, \varepsilon)$ soit fini pour $\varepsilon>0$. Il suffit de poser $\widetilde{\Phi}(K, \varepsilon)=\bar{A} \cup \varphi(K, \varepsilon)$.

Affirmation $\widetilde{2}$. Il existe une fonction continue $\widetilde{\Psi}: Q \times] 0, \eta] \rightarrow 2_{A}^{I}$ vérifiant

$$
\begin{gathered}
\left.\left.\widetilde{\Psi}^{-1}\left(\mathcal{N}_{A}\right)=C \times\right] 0, \eta\right], \\
\varrho(\bar{A}, \widetilde{\Psi}(q, \varepsilon)) \leq 2 \varepsilon \quad \forall(q, \varepsilon) \in C \times] 0, \eta],
\end{gathered}
$$

( $\widetilde{9}) \quad a+2 \varepsilon$ est la borne supérieure de l'intérieur de $\widetilde{\Psi}(q, c) \cap[a, a+3 \eta]$, 
( $\widetilde{10})$ si les intérieurs des ensembles $\widetilde{\Psi}(q, \varepsilon) \cap[a+\varepsilon, a+2 \varepsilon]$ et $\widetilde{\Psi}\left(q^{\prime}, \varepsilon\right) \cap$ $[a+\varepsilon, a+2 \varepsilon]$ sont égaux, alors $q=q^{\prime}$.

Nous commencerons par construire une fonction continue $\psi: Q \times] 0, \eta]$ $\rightarrow 2_{A}^{I}$ vérifiant

$$
\begin{gathered}
\left.\left.\psi(q, \varepsilon) \subset N_{\varepsilon} \quad \forall(q, \varepsilon) \in Q \times\right] 0, \eta\right], \\
\left.\left.\psi^{-1}\left(\mathcal{N}_{A}\right)=C \times\right] 0, \eta\right] .
\end{gathered}
$$

Pour cela, soit $\left\{L_{n}\right\}_{n=1}^{\infty}$ une énumération des composantes de $I \backslash \bar{A}$. Pour $\varepsilon \geq 0$, soit $P_{n}(\varepsilon)=\left\{t \in \bar{L}_{n}: d(t, A)=\varepsilon\right\}$; cet ensemble contient au plus deux points. Puisque $\xi(q)$ contient $\bar{A}$, la fonction $\xi_{n}: Q \rightarrow 2^{I}$ définie par $\xi_{n}(q)=\xi(q) \cap \bar{L}_{n}$ est continue, donc il en est de même de la fonction $\mu_{n}: Q \rightarrow I$ définie par

$$
\mu_{n}(q)=\sup \left\{d(t, \bar{A}): t \in \xi_{n}(q)\right\} .
$$

Définissons une fonction $\left.\left.\psi_{n}: Q \times\right] 0, \eta\right] \rightarrow 2^{I}$ par

$$
\psi_{n}(q, \varepsilon)= \begin{cases}\xi_{n}(q) & \text { si } \mu_{n}(q) \leq \varepsilon / 2, \\ \xi_{n}(q) \cup P_{n}\left(2 \mu_{n}(q)-\varepsilon\right) & \text { si } \varepsilon / 2 \leq \mu_{n}(q), \\ \left(\xi_{n}(q) \cap N_{\varepsilon}\right) \cup P_{n}(\varepsilon) & \text { si } \varepsilon \leq \mu_{n}(q) .\end{cases}
$$

Il est facile de voir que cette définition a un sens et que $\psi_{n}$ est continue. En outre, $\psi_{n}(q, \varepsilon)$ est contenu dans $N_{\varepsilon}$, donc il en est de même de l'ensemble

$$
\psi(q, \varepsilon)=\bar{A} \cup \bigcup_{n=1}^{\infty} \psi_{n}(q, \varepsilon) .
$$

Puisque les $\psi_{n}(q, \varepsilon)$ sont des compacts, $\psi(q, \varepsilon)$ aussi, et la continuité des $\psi_{n}, n \geq 1$, entraîne celle de $\psi$. Remarquons que, pour tout $n, \psi_{n}(q, \varepsilon)$ est la réunion de $\xi_{n}(q) \cap N_{\varepsilon}$ et d'au plus deux points, et que $\psi_{n}(q, \varepsilon)=\xi_{n}(q)$ si le diamètre de $L_{n}$ est inférieur à $\varepsilon / 2$, ce qui est le cas de toutes les $L_{n}$ sauf un nombre fini. Par suite, $\psi(q, \varepsilon)$ est la réunion de $\xi(q) \cap N_{\varepsilon}$ et d'un ensemble fini, donc (17) résulte de (1).

Définissons une fonction $\widetilde{\theta}: Q \rightarrow 2^{I}$ en posant, pour $q=\left(q_{n}\right)$ dans $Q$,

$$
\widetilde{\theta}(q)=\{0\} \cup[1 / 2,1] \cup \bigcup_{n=1}^{\infty}\left[2^{-(n+1)}, 2^{-(n+1)}+q_{n} \cdot 2^{-(n+2)}\right] .
$$

Il est clair que $\widetilde{\theta}$ est continue et que si $q$ et $q^{\prime}$ sont deux points distincts de $Q$, alors les intérieurs des ensembles $\widetilde{\theta}(q)$ et $\widetilde{\theta}\left(q^{\prime}\right)$ sont distincts. Pour $0<\varepsilon \leq \eta$, soit $u_{\varepsilon}$ l'application affine de $\mathbb{R}$ dans $\mathbb{R}$ envoyant 0 sur $a+\varepsilon$ et 1 sur $a+2 \varepsilon$. Posons enfin

$$
\widetilde{\Psi}(q, \varepsilon)=\psi(q, \varepsilon) \cup u_{\varepsilon}(\widetilde{\theta}(q)) .
$$

Cette fonction est évidemment continue et, d'après (3) et (16), nous avons $\bar{A} \subset \widetilde{\Psi}(q, \varepsilon) \subset N_{2 \varepsilon}$, d'où $(\widetilde{8})$. La condition $(\widetilde{7})$ résulte de (16) et de 
l'égalité $\widetilde{\Psi}(q, \varepsilon) \cap N_{\varepsilon}=\psi(q, \varepsilon) \cup\{a+\varepsilon\}$, tandis que $(\widetilde{9})$ et ( $\left.\widetilde{10}\right)$ résultent de la construction de $\widetilde{\theta}$ et du fait que $\widetilde{\Psi}(q, \varepsilon) \cap[a+\varepsilon, a+3 \eta]=u_{\varepsilon}(\widetilde{\theta}(q))$.

La démonstration s'achève maintenant comme pour $\mathcal{L}_{A}$. Nous choisissons une fonction continue $\varepsilon: U \rightarrow] 0, \eta]$ vérifiant (14) et

$$
\varrho(K, \widetilde{\Phi}(K, \varepsilon(K)))<\omega(K) .
$$

Posant $\widetilde{\varepsilon}(c)=\varepsilon(F(c))$, nous définissons $G$ par

$$
G(c)=\widetilde{\Phi}(F(c), \widetilde{\varepsilon}(c)) \cup \widetilde{\Psi}(c, \widetilde{\varepsilon}(c)) .
$$

D'après $(7), G(c)$ appartient à $\mathcal{N}_{A}$ et, en utilisant $(\widetilde{13}),(\widetilde{8})$ et le fait que $\bar{A} \subset F(c) \cap \widetilde{\Psi}(c, \widetilde{\varepsilon}(c))$, on établit facilement que

$$
\varrho(F(c), G(c)) \leq 2 \omega(F(c)),
$$

ce qui entraîne que $G$ est $\mathcal{U}$-proche de $F$. Il résulte de (15) que les ensembles $G(c) \cap[a, a+3 \eta]$ et $\widetilde{\Psi}(c, \widetilde{\varepsilon}(c)) \cap[a, a+3 \eta]$ ont le même intérieur. L'injectivité de $G$ résulte alors de $(\widetilde{9})$ et $(\widetilde{10})$.

Pour voir que $G$ est un plongement fermé dans $U$, soit $\left\{c_{n}\right\}_{n=1}^{\infty}$ une suite de points de $C$ telle que la suite $\left\{G\left(c_{n}\right)\right\}$ converge vers un élément $K$ de $U$. Posant $\varepsilon_{n}=\widetilde{\varepsilon}\left(c_{n}\right)$, nous pouvons supposer que $\left\{\varepsilon_{n}\right\}_{n=1}^{\infty}$ converge vers $\varepsilon_{0} \in[0, \eta]$, que $\left\{c_{n}\right\}$ converge vers un point $c_{0}$ de $Q$ et que $\left\{F\left(c_{n}\right)\right\}$ converge vers $F_{0} \in 2_{A}^{I}$. Si $\varepsilon_{0}=0,(\widetilde{4})$ et $(\widetilde{8})$ nous donnent, en passant à la limite dans la définition de $G, K=F_{0} \cup \bar{A}=F_{0}$, donc $F_{0}$ appartient à $U$ et $\left\{\varepsilon_{n}\right\}$ tend vers $\varepsilon\left(F_{0}\right)>0$, ce qui est contradictoire. Si $\varepsilon_{0}>0$, nous avons

$$
K=\widetilde{\Phi}\left(F_{0}, \varepsilon_{0}\right) \cup \widetilde{\Psi}\left(c_{0}, \varepsilon_{0}\right),
$$

et, puisque $K$ appartient à $\mathcal{N}_{A}$, il en est de même de $\widetilde{\Psi}\left(c_{0}, \varepsilon_{0}\right)$ d'après $(15)$, donc $c_{0}$ appartient à $C$ d'après $(\widetilde{7})$.

Pour montrer que $G(C)$ est un $Z$-ensemble dans $U$, il suffit de construire une fonction continue $\widetilde{\Lambda}: \mathcal{N}_{A} \times[0, \eta] \rightarrow \mathcal{N}_{A}$ vérifiant $\widetilde{\Lambda}(K, 0)=K$ et $\left.\left.\widetilde{\Lambda}\left(\mathcal{N}_{A} \times\right] 0, \eta\right]\right) \cap G(C)=\emptyset$, ce qui peut se faire comme suit. Soit $M=$ $\{1\} \cup \bigcup_{n=1}^{\infty}\left[1-2^{-n}, 1-\left(2^{-n}-2^{-(n+2)}\right)\right]$. Posons

$$
\begin{aligned}
& \widetilde{\Lambda}(K, 0)=K, \\
& \widetilde{\Lambda}(K, \varepsilon)=\widetilde{\Phi}(K, \varepsilon) \cup N_{\varepsilon} \cup u_{\varepsilon}(M), \quad 0<\varepsilon \leq \eta .
\end{aligned}
$$

La continuité de $\widetilde{\Lambda}$ se vérifie facilement. Pour voir que $\left.\left.\widetilde{\Lambda}\left(\mathcal{N}_{A} \times\right] 0, \eta\right]\right) \cap$ $G(C)=\emptyset$, il suffit d'observer que, pour $\varepsilon>0$, l'ensemble des composantes de l'intérieur de $\widetilde{\Lambda}(K, \varepsilon) \cap[a, a+3 \eta]$ n'a pas de plus grand élément tandis que l'ensemble des composantes de l'intérieur de $G(c) \cap[a, a+3 \eta]$ a pour plus grand élément $u_{\tilde{\varepsilon}(c)}(11 / 2,1[)$. 
3. Démonstration du lemme 2. Nous noterons $\mathbb{N}$ l'ensemble des entiers $>0, \mathbb{N}^{*}$ l'ensemble des suites finies (non vides) d'entiers $>0$ et $J$ l'ensemble des suites infinies d'entiers $>0$. Pour $\sigma$ dans $\mathbb{N}^{*}$, nous noterons $|\sigma|$ la longueur de la suite $\sigma$. Si $\sigma=\left\langle s_{1}, \ldots, s_{k}\right\rangle$ est une suite de longueur $k$ dans $\mathbb{N}^{*}$ et $p$ un entier $>0$, nous noterons $\langle\sigma, p\rangle$ la suite $\left\langle s_{1}, \ldots, s_{k}, p\right\rangle$ de longueur $k+1$. Si $\sigma$ appartient à $J$ et $n$ est un entier $>0$ ou si $\sigma$ appartient à $\mathbb{N}^{*}$ et $n$ est un entier $\leq|\sigma|$, nous noterons $\sigma \mid n$ la suite des $n$ premiers termes de $\sigma$. Soit $\tau$ un élément de $\mathbb{N}^{*}$; si $\sigma$ appartient à $J$ ou si $\sigma$ appartient à $\mathbb{N}^{*}$ et vérifie $|\sigma|>|\tau|$, nous écrivons $\tau<\sigma$ s'il existe un entier $n$ tel que $\tau=\sigma \mid n$. Nous noterons 0 la suite vide, de sorte que $0<\sigma$ pour tout $\sigma$ dans $\mathbb{N}^{*}$.

Soit $\mathcal{F}(X)$ l'ensemble des fermés de $X$. Puisque $F$ est analytique, il y a (voir [8], §35, II) une fonction $S: \mathbb{N}^{*} \rightarrow \mathcal{F}(X)$ vérifiant

$$
F=\bigcup_{\sigma \in J} \bigcap_{n=1}^{\infty} S(\sigma \mid n) .
$$

D'après le lemme 1.5 de [3], nous pouvons supposer que

$$
S(\tau) \subset \operatorname{Int} S\left(\tau^{\prime}\right) \quad \text { si } \tau^{\prime}<\tau .
$$

Pour $\sigma$ dans $\mathbb{N}^{*}$, prenons une fonction continue $\lambda_{\sigma}$ de $X$ dans $I$ vérifiant

$$
\begin{array}{lll}
\lambda_{\sigma}(x)=1 & \text { si } x \in S(\sigma), \\
\lambda_{\sigma}(x)=0 & \text { si } x \notin S(\sigma \mid n-1) & \text { pour }|\sigma|=n \geq 2 .
\end{array}
$$

Nous aurons besoin du lemme suivant :

Lemme 3. Il existe un fermé $C$ de I vérifiant

(i) $E=C \cap A$ est homéomorphe à l'ensemble des irrationnels,

(ii) $D=C \backslash A$ est homéomorphe à $\mathbb{Q}$,

(iii) $\bar{D}=C$.

Ce lemme est un cas particulier d'un théorème d'Hurewicz [7], dont une démonstration se trouve aussi dans l'article de F. Topsøe et J. HoffmannJørgensen dans [9] (théorème 1.4.2, p. 333).

Puisque $A$ est non dense, $C$ est un ensemble de Cantor. Soit $\left\{d_{n}\right\}_{n=1}^{\infty}$ une énumération de $D$. Nous allons construire, par récurrence sur $|\sigma|$, des sousintervalles $I(\sigma)=[a(\sigma), b(\sigma)]\left(\sigma \in\{0\} \cup \mathbb{N}^{*}\right)$ de façon que, notant $\stackrel{\circ}{I}(\sigma)=$ ]$a(\sigma), b(\sigma)\left[\right.$ et $J_{\sigma}=\{a(\sigma), b(\sigma)\} \cup \bigcup_{p=1}^{\infty} I(\langle\sigma, p\rangle)$, les conditions suivantes soient vérifiées.

(5) $\quad$ Si $\sigma \neq \sigma^{\prime}$ et $|\sigma|=\left|\sigma^{\prime}\right|$, alors $I(\sigma) \cap I\left(\sigma^{\prime}\right)=\emptyset$.

(6) $\quad$ Si $\sigma<\tau$, alors $I(\tau) \subset \stackrel{\circ}{I}(\sigma)$.

(7) $\quad \delta(I(\sigma)) \leq 2^{-|\sigma|}$.

(8) $J_{\sigma}$ est fermé. 
(9) $\quad a(\sigma)$ et $b(\sigma)$ appartiennent à $D$.

(10) Le premier élément de $D \cap \stackrel{\circ}{I}(\sigma)$ (pour l'énumération fixée de $D$ ) n'appartient pas à $J_{\sigma}$.

(11) Ou bien $a(\sigma)$ n'est pas l'extrémité gauche d'un intervalle de $I \backslash C$, ou bien $b(\sigma)$ n'est pas l'extrémité droite d'un tel intervalle.

Soient $i_{0}<j_{0}$ deux points de $D$ tels que $] i_{0}, j_{0}\left[\cap C \neq \emptyset\right.$. Si $i_{0}$ n'est pas l'extrémité gauche d'un intervalle de $I \backslash C$ ou si $j_{0}$ n'est pas l'extrémité droite d'un tel intervalle, prenons $I(0)=\left[i_{0}, j_{0}\right]$. Sinon, soit $k_{0}$ un point de $D$ tel que $i_{0}<k_{0}<j_{0}$. Si $k_{0}$ n'est pas l'extrémité droite d'un intervalle de $I \backslash C$, prenons $I(0)=\left[i_{0}, k_{0}\right]$; dans le cas contraire, $k_{0}$ ne peut pas être aussi l'extrémité gauche d'un intervalle de $I \backslash C$, et nous prenons $I(0)=\left[k_{0}, j_{0}\right]$.

Supposons les $I(\tau)$ construits quand $|\tau|=k$, et soit $\sigma$ de longueur $k$. Supposons que $b(\sigma)$ ne soit pas l'extrémité droite d'un intervalle de $I \backslash C$. Nous pouvons alors trouver deux suites croissantes $\left\{i_{p}\right\},\left\{j_{p}\right\}$ de points de $D \cap \stackrel{i}{I}(\sigma)$ convergeant vers $b(\sigma)$, telles que $i_{p}<j_{p}<i_{p+1}$ et que $] i_{p}, j_{p}[\cap C$ $\neq \emptyset$ pour tout $p$. Nous supposons aussi $i_{1}$ choisi de façon que $b(\sigma)-i_{1}<$ $2^{-(k+1)}$ et que $\left[i_{1}, b(\sigma)\right]$ ne contienne pas le premier élément de $D \cap \stackrel{\circ}{I}(\sigma)$. Si $i_{p}$ n'est pas l'extrémité gauche d'un intervalle de $I \backslash C$ ou si $j_{p}$ n'est pas l'extrémité droite d'un tel intervalle, posons $I(\langle\sigma, p\rangle)=\left[i_{p}, j_{p}\right]$. Sinon, prenons un point $k_{p}$ de $D$ vérifiant $i_{p}<k_{p}<j_{p}$ et prenons $I(\langle\sigma, p\rangle)=\left[i_{p}, k_{p}\right]$ ou $\left[k_{p}, j_{p}\right]$ selon que $k_{p}$ n'est pas ou est l'extrémité droite d'un intervalle de $I \backslash$ C. Il est alors facile de vérifier les conditions (5) à (11). Si $b(\sigma)$ est l'extrémité droite d'un intervalle de $I i \backslash C$, alors $a(\sigma)$ n'est pas l'extrémité gauche d'un tel intervalle et nous procédons de façon analogue, mais en prenant pour $\left\{i_{p}\right\}$ et $\left\{j_{p}\right\}$ des suites décroissantes tendant vers $a(\sigma)$ et vérifiant $j_{p+1}<i_{p}<j_{p}$.

Remarquons que

$\forall \sigma \in J, \bigcap_{n=1}^{\infty} I(\sigma \mid n)$ contient exactement un point $e_{\sigma}$, qui appartient à $E$.

En effet, (6) et (7) entraînent que $\bigcap_{n=1}^{\infty} I(\sigma \mid n)$ contient exactement un point $e_{\sigma} ;(11)$ entraîne que $I(\sigma \mid n) \cap C \neq \emptyset$ pour tout $n$, donc $e_{\sigma}$ appartient à $C$. Enfin, $e_{\sigma}$ ne peut appartenir à $D$, car cela contredirait la condition (10) pour $I(\sigma \mid n)$ si $n$ est assez grand.

Nous allons maintenant construire, pour tout $\sigma$ dans $\mathbb{N}^{*}$, une suite $\left\{K_{\sigma}^{n}\right\}_{n=1}^{\infty}$ d'intervalles deux à deux disjoints de façon que, posant $L_{\sigma}=$ $J_{\sigma} \cup \bigcup_{n=1}^{\infty} K_{\sigma}^{n}$, les conditions suivantes soient vérifiées :

$$
\begin{gathered}
K_{\sigma}^{n} \subset I(\sigma) \backslash J_{\sigma}, \\
L_{\sigma} \text { est fermé, } \\
A \cap I(\sigma) \subset L_{\sigma},
\end{gathered}
$$

les extrémités de $K_{\sigma}^{n}$ n'appartiennent pas à $A$. 
Fixons $\sigma$ dans $\mathbb{N}^{*}$. Soient $\left.Q_{p}=\right] c_{p}, d_{p}[, p \geq 1$, les composantes de $I(\sigma) \backslash J_{\sigma}$ (il y en a une infinité d'après (5) et (6)). Puisque $A$ est non dense, nous pouvons trouver dans $Q_{p}$ deux suites $\left\{y_{p}^{r}\right\}_{r=1}^{\infty}$ et $\left\{z_{p}^{r}\right\}_{r=1}^{\infty}$ convergeant vers $d_{p}$ et deux suites $\left\{u_{p}^{r}\right\}_{r=1}^{\infty}$ et $\left\{v_{p}^{r}\right\}_{r=1}^{\infty}$ convergeant vers $c_{p}$ de façon que, pour tout $p$,

$$
v_{p}^{r+1}<u_{p}^{r}<v_{p}^{r} \leq v_{p}^{1}<y_{p}^{1} \leq y_{p}^{r}<z_{p}^{r}<y_{p}^{r+1},
$$

et que $\left[u_{p}^{r}, v_{p}^{r}\right] \cap A=\emptyset=\left[y_{p}^{r}, z_{p}^{r}\right] \cap A$. Il suffit alors de prendre pour $K_{\sigma}^{n}$, $n \geq 1$, une énumération des composantes de $I(\sigma) \backslash\left(J_{\sigma} \cup\left(\bigcup_{p, r=1}^{\infty}\right] u_{p}^{r}, v_{p}^{r}[\cup\right.$ ]$\left.y_{p}^{r}, z_{p}^{r}[)\right)$. Remarquons que, pour tout $\sigma$, l'ensemble $I(\sigma) \backslash L_{\sigma}$ n'est pas vide.

Pour $\sigma$ dans $\mathbb{N}^{*}$, prenons une fonction continue $\psi_{\sigma}: I \rightarrow 2^{I}$ vérifiant

$$
\psi_{\sigma}(0)=I \supset \psi_{\sigma}(t) \supset \psi_{\sigma}(1)=(I \backslash \stackrel{\circ}{I}(\sigma)) \cup L_{\sigma}, \quad 0 \leq t \leq 1 .
$$

D'après (15), $\psi_{\sigma}(t)$ contient $A$, donc la fonction $\xi_{\sigma}=\psi_{\sigma} \circ \lambda_{\sigma}$ envoie $X$ dans $2_{A}^{I}$. Par suite, pour $x$ dans $X$, l'ensemble

$$
\xi(x)=\bigcap_{\sigma \in \mathbb{N}^{*}} \xi_{\sigma}(x)
$$

appartient à $2_{A}^{I}$. Il résulte de $(5)$ et des définitions de $J_{\sigma}$ et $L_{\sigma}$ que les ouverts $\stackrel{\circ}{I}(\sigma) \backslash L_{\sigma}, \sigma \in \mathbb{N}^{*}$, sont deux à deux disjoints; comme, pour tout $\sigma$, $\xi_{\sigma}(x)$ contient le complémentaire de $\stackrel{\circ}{I}(\sigma) \backslash L_{\sigma}$, la continuité des $\xi_{\sigma}$ entraîne celle de $\xi$.

Soit $x$ un point de $F$. D'après (1), il y a un $\tau$ dans $J$ tel que $x \in$ $\bigcap_{n=1}^{\infty} S(\tau \mid n)$. D'après (6) et $(12),\{I(\tau \mid n)\}_{n=1}^{\infty}$ est une base de voisinage du point $e_{\tau}$ de $A$. D'après $(3), \lambda_{\tau \mid n}(x)=1$, donc, d'après $(17),\left(\stackrel{\circ}{I}(\tau \mid n) \backslash L_{\tau \mid n}\right) \cap$ $\xi(x)=\emptyset$, ce qui montre que $\xi(x)$ ne contient aucun voisinage de $e_{\tau}$, donc que $\xi(x) \notin \mathcal{N}_{A}$.

Pour achever de prouver que $\xi^{-1}\left(\mathcal{N}_{A}\right)=X \backslash F$, il reste à montrer que si $x$ est un point de $X \backslash F$ et $t$ un point de $A$, alors $\xi(x)$ est un voisinage de $a$. D'après (5), pour tout $k, t$ appartient à au plus un $I(\sigma)$ où $\sigma$ est de longueur $k$; si $t$ appartient à $I(\sigma)$, alors, pour tout $m<|\sigma|, t$ appartient à $I(\sigma \mid m)$. D'après (6), si $t$ appartient à $I(\sigma)$, alors, pour tout $m<|\sigma|, t$ appartient à $I(\sigma \mid m)$.

(a) $t$ n'appartient à aucun $I(\langle p\rangle), p \geq 1$.

D'après (9), $t$ n'appartient pas à $J_{0}$, donc $I \backslash J_{0}$ est un voisinage de $t$ contenu dans tous les $\xi_{\sigma}(x)$, donc aussi dans $\xi(x)$.

(b) Il existe un $k$ et un $\tau$ de longueur $k$ tels que $t$ appartienne à $I(\tau)$ mais pas à $\bigcup_{|\sigma|=k+1} I(\sigma)$.

Alors, $t$ n'appartient pas à $J_{\tau}$, donc, d'après (15) et (16), il y a un entier $n$ tel que $K_{\tau}^{n}$ soit un voisinage de $t$. Il suffit de montrer que tous les $\xi_{\sigma}(x)$ 
contiennent $K_{\tau}^{n}$. C'est clair si $\sigma=\tau$ puisque $K_{\tau}^{n} \subset L_{\tau}$. Soit $\sigma \neq \tau$ et soit $m=|\sigma|$. Si $m=k$ ou si $m<k$ et $\sigma \neq \tau \mid m$ ou si $m>k$ et $\tau \neq \sigma \mid k$, il résulte de (5) et (6) que $I(\sigma) \cap K_{\tau}^{n} \subset I(\sigma) \cap I(\tau)=\emptyset$, donc $\xi_{\sigma}(x) \supset K_{\tau}^{n}$. Si $m<k$ et $\sigma=\tau \mid m$, alors $K_{\tau}^{n} \subset I(\tau) \subset J_{\sigma}$ d'après (6), donc $\xi_{\sigma}(x) \supset K_{\tau}^{n}$. Si $m>k$ et $\tau=\sigma \mid k$, alors $I(\sigma) \subset J_{\tau}$ d'après (6), donc $I(\sigma) \cap K_{\tau}^{n}=\emptyset$ d'après (13), d'où encore $\xi_{\sigma}(x) \supset K_{\tau}^{n}$.

(c) Il existe un unique $\tau \in J$ tel que $t \in \bigcap_{n=1}^{\infty} I(\tau \mid n)$.

D'après (1), il y a un $n>1$ tel que $x \notin S(\tau \mid n-1)$. D'après (6), $I(\tau \mid n)$ est un voisinage de $t$; il suffit donc de montrer que tous les $\xi_{\sigma}(x)$ contiennent $I(\tau \mid n)$. Soit $k=|\sigma|$. Si $k<n$ et si $\sigma \neq \tau \mid k$, alors $I(\sigma) \cap I(\tau \mid n) \subset$ $I(\sigma) \cap I(\tau \mid k)=\emptyset$; si $\sigma=\tau \mid k, I(\tau \mid n) \subset J_{\sigma}$ d'après (6); dans les deux cas, $I(\tau \mid n) \subset \xi_{\sigma}(x)$. Si $k=n$ et $\sigma \neq \tau \mid n$ ou si $k>n$ et $\sigma|n \neq \tau| n$, alors $I(\sigma) \cap I(\tau \mid n)=\emptyset$ d'après (5) et (6), donc $I(\tau \mid n) \subset \xi_{\sigma}(x)$. Enfin, si $\sigma=\tau \mid n$ ou si $k>n$ et $\sigma|n=\tau| n$, alors $\sigma|n-1=\tau| n-1$, donc $\lambda_{\sigma}(x)=0$ d'après (4) et $(2)$ et $\xi_{\sigma}(x)=I$ d'après (17), d'où le résultat.

\section{Bibliographie}

[1] S. Banach et C. Kuratowski, Sur la structure des ensembles linéaires, Studia Math. 4 (1933), 95-99.

[2] M. Bestvina and J. Mogilski, Characterizing certain incomplete infinite-dimensional absolute retracts, Michigan Math. J. 33 (1986), 291-313.

[3] R. Cauty, Caractérisation topologique de l'espace des fonctions dérivables, Fund. Math. 138 (1991), 35-58.

[4] R. Cauty, T. Dobrowolski and W. Marciszewski, A contribution to the topological classification of the spaces $C_{\mathrm{p}}(X)$, ibid. 142 (1993), 269-301.

[5] D. Curtis and Nguyen To Nhu, Hyperspaces of finite subsets which are homeomorphic to $\aleph_{0}$-dimensional linear metric spaces, Topology Appl. 19 (1985), 251260.

[6] D. Curtis and R. M. Schori, Hyperspaces of Peano continua are Hilbert cubes, Fund. Math. 101 (1978), 19-38.

[7] W. Hurewicz, Relative perfekte Teile von Punktmengen und Mengen (A), ibid. 12 (1928), 78-109.

[8] C. Kuratowski, Topologie I, 4ème édition, PWN, Warszawa, 1958.

[9] C. A. Rogers et al., Analytic Sets, Academic Press, London, 1980.

[10] H. Toruńczyk, Concerning locally homotopy negligible sets and characterization of $l_{2}$-manifolds, Fund. Math. 101 (1978), 93-110.

22, RUE JOUVENET

75016 PARIS, FRANCE 